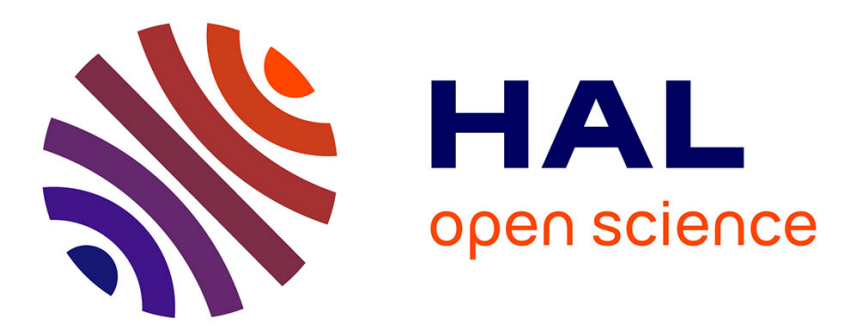

\title{
Combined Laplacian-equivolumic model for studying cortical lamination with ultra high field MRI (7 T)
}

Yann Leprince, Fabrice Poupon, Thierry Delzescaux, Dominique Hasboun, Cyril Poupon, Denis Rivière

\section{- To cite this version:}

Yann Leprince, Fabrice Poupon, Thierry Delzescaux, Dominique Hasboun, Cyril Poupon, et al.. Combined Laplacian-equivolumic model for studying cortical lamination with ultra high field MRI (7 T). 2015 IEEE 12th International Symposium on Biomedical Imaging (ISBI), IEEE, Apr 2015, New York, United States. pp.580-583, 10.1109/ISBI.2015.7163940 • cea-01119475v2

\section{HAL Id: cea-01119475 \\ https://hal-cea.archives-ouvertes.fr/cea-01119475v2}

Submitted on 18 Aug 2015

HAL is a multi-disciplinary open access archive for the deposit and dissemination of scientific research documents, whether they are published or not. The documents may come from teaching and research institutions in France or abroad, or from public or private research centers.
L'archive ouverte pluridisciplinaire HAL, est destinée au dépôt et à la diffusion de documents scientifiques de niveau recherche, publiés ou non, émanant des établissements d'enseignement et de recherche français ou étrangers, des laboratoires publics ou privés. 


\title{
COMBINED LAPLACIAN-EQUIVOLUMIC MODEL FOR STUDYING CORTICAL LAMINATION WITH ULTRA HIGH FIELD MRI (7 T)
}

\author{
Yann Leprince $e^{1,2}$ \\ Dominique Hasboun ${ }^{4}$ \\ Fabrice Poupon ${ }^{1}$ \\ Cyril Poupon ${ }^{1}$ \\ Thierry Delzescaux ${ }^{3}$
Denis Rivière \\ ${ }^{1}$ NeuroSpin, CEA, Gif-sur-Yvette, France \\ ${ }^{2}$ Université Paris-Sud, Orsay, France \\ 3 MIRCen, CEA, Fontenay-aux-Roses, France \\ ${ }^{4}$ Université Pierre et Marie Curie, Paris, France
}

\begin{abstract}
The fine spatial resolution and novel contrasts offered by highfield magnetic resonance allow in vivo detection of histological layers in the cerebral cortex. This opens the way to in vivo analysis of cortical lamination, but the comparison of lamination profiles has proved challenging because the layers' geometry is strongly influenced by cortical curvature. This paper introduces a model of the micro-structural organization of the cortex, which can compensate for the effect of cortical curvature. Layers are modelled by an equivolumic principle, while the vertical structure of the cortex is represented with a Laplacian model. In this framework, lamination profiles can be represented in a way that preserves the original voxel sampling of the acquisition. This model is validated on a magnetic resonance image of a post-mortem human brain acquired on a human $7 \mathrm{~T}$ scanner at $0.35 \mathrm{~mm}$ resolution.
\end{abstract}

Index Terms - cortex, layer, lamination, column, equivolumic, Laplace, Bok, cytoarchitecture, high-field MRI, brain, advection

\section{INTRODUCTION}

High-field magnetic resonance imaging (MRI) at $7 \mathrm{~T}$ and beyond opens a window into the study of brain micro-architecture. In particular, the layers of different histological compositions that make up the cortex can now be imaged with resolutions better than $0.5 \mathrm{~mm}$. By enabling a better characterization of the cortical lamination, this work constitutes a step towards the automated detection of cytoarchitectural brain regions, which are currently accessible only through post-mortem histology or architectural atlases such as Brodmann's. In vivo reconstruction of subject-specific architectonic parcellations will be an exciting tool for the study of brain morphology and function.

The convoluted geometry of the cortex poses a challenge to the automatic characterization of cortical layers, because their shape is influenced by cortical curvature (see Fig. 1). As a result, robust extraction of lamination profiles requires modelling

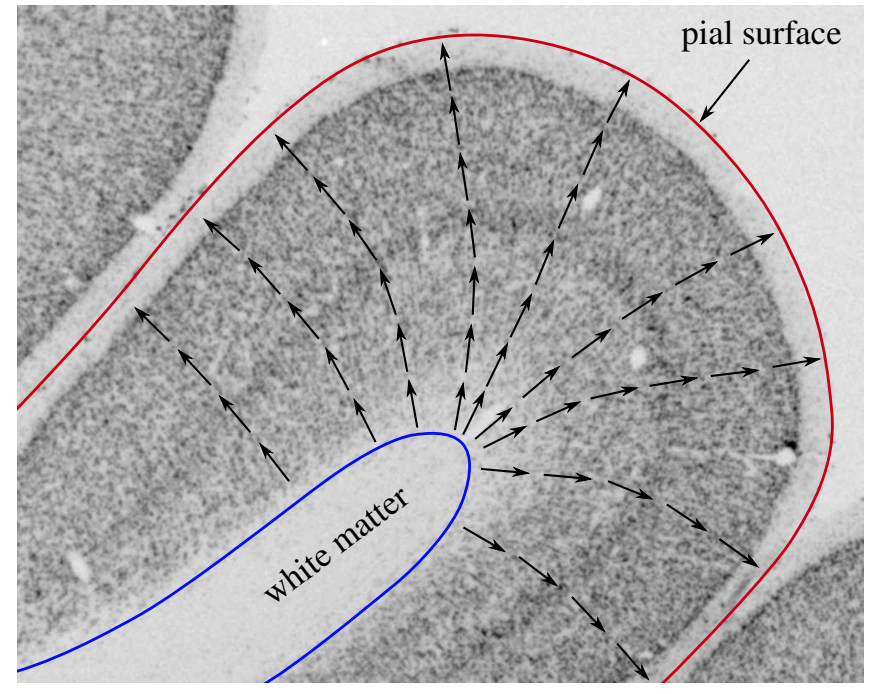

Fig. 1: NeuN-stained histological section of a macaque brain showing a gyral crown, with visible cortical lamination. Arrows show the directions given by the 3D Laplacian model of cortical columns (vector field $\vec{F}$, see equation 2).

the shape of cortical layers. In addition, the vertical structure of the cortex, which is represented by cortical columns, is also influenced by curvature. The term "cortical columns" is used here in a weak sense, to refer to the vertical alignment of neurons which appears on cell-body-stained images of the cortex such as Fig. 1. It is not meant to refer to functional or anatomical entities of defined horizontal extent.

A geometric model of cortical columns and layers is proposed in this paper, which can be used to extract lamination profiles which are robust to cortical curvature.

\section{LAPLACIAN MODEL OF CORTICAL COLUMNS}

While they are critical to the vertical structure of the cortex, cortical columns cannot be directly imaged with MRI. What 
can be measured, on the other hand, is the geometry of the cortex, in the form of a segmentation derived from an anatomical image. A Laplacian model can then be used to infer the shape of cortical columns from the geometry of the cortex.

A voxel-wise classification of the brain is used as a starting point, where each voxel is assigned to GM (grey matter), WM (white matter), or CSF (cerebrospinal fluid). The Laplacian field is obtained by solving the Laplace equation (1) for $T$ within cortical grey matter, with constant values imposed on the exterior: $T_{\mathrm{CSF}}=0$, and $T_{\mathrm{WM}}=1$. The orientation of cortical columns in each point of the cortex is then represented by the normalized gradient $\vec{F}$, defined by (2).

$$
\begin{gathered}
\frac{\partial^{2} T}{\partial x^{2}}+\frac{\partial^{2} T}{\partial y^{2}}+\frac{\partial^{2} T}{\partial z^{2}}=0 \\
\vec{F}=\frac{\nabla T}{\|\nabla T\|}
\end{gathered}
$$

This Laplacian model was introduced [1] for measuring cortical thickness on $3 \mathrm{D}$ magnetic resonance images. It was introduced later in the context of 2D cytoarchitecture, under an electrodynamic formalism [2]. In both cases, the Laplacian model is used to construct curved cortical traverses that follow $\vec{F}$.

The accuracy of the Laplacian model of columns has not been formally assessed. However, previous evidence [3, figure 2] suggests that the direction of cortical columns is well represented by the Laplacian model, on a 2D histological slice orthogonal to the cortex. Figure 1 also shows good agreement between the direction of columns visible on a non-orthogonal histological slice, and their Laplacian model $\vec{F}$ based on a 3D segmentation built from serial photographic images of the sample [4].

\section{EQUIVOLUMIC CORTICAL DEPTH METRIC}

Lamination can be characterized with cortical profiles, which represent the evolution of a parameter (e.g. MR signal) as a function of depth. However, the very definition of depth poses a challenge, because the curvature of the cortex induces variations in the thickness of cortical layers. Such variations are induced by the presence of sulci and gyri, and should not be mistaken as boundaries between architectural regions.

As a result, the intra-cortical depth should be measured in a way that is robust to the effect of curvature. In other words, a good measure of depth should be constant across sulci and gyri for a given layer within an architecturally homogeneous region.

\subsection{Equivolumic principle}

A model of cortical layers was proposed recently [5], based on the following observation: when considering vertical cortical segments separated by cortical columns, the volume of a given layer is similar in neighbouring cortical segments [6]. This principle, which can be termed equivolumic, results in thicker layers in regions of high curvature where columns are squeezed, because conservation of volume is achieved by extending the layer vertically.

For instance in gyral crowns (see Fig. 1), cortical columns are squeezed tightly in deep layers whereas they spread towards superficial layers; as a result deep layers appear thicker, and superficial layers appear thinner. The reverse happens in sulcal fundi, where the curvature is inverted.

Wähnert and collaborators [5] present an implementation of the equivolumic principle based on the estimation of intracortical surfaces corresponding to cortical layers, in a level set framework. The shape of columns is approximated by the normal to equidistant laminae, and by linear interpolation of the curvature of the pial and grey-white surfaces.

\subsection{Proposed implementation}

The proposed method implements an equivolumic metric based on Bok's observation and the Laplacian model of cortical columns, within an advection formalism. The squeezing of cortical columns is estimated directly from the divergence of $\vec{F}$, and the shape of whole cortical columns is taken into account using the advection of an elementary surface along $\vec{F}$.

This method is based on the advection (transportation) of each intra-cortical point along $\vec{F}$. The advection path can be followed iteratively using Euler's method according to (3), where the current point $M_{n}$ is transported to $M_{n+1}$ with a step size $\delta l$.

$$
M_{n+1}=M_{n}+\delta l \vec{F}\left(M_{n}\right)
$$

Starting from each cortical voxel $M$, an elementary surface $\delta S$ is considered around $M$ (see Fig. 2). As $M$ is advected along the vector field $\vec{F}$, the associated surface element $\delta S$ changes area, as it undergoes dilation or compression according to the divergence of $\vec{F}$. Specifically, at each step $n$ of the advection, the elementary surface $\delta S_{n}$ evolves into $\delta S_{n+1}$, according to (4), where $\delta l$ is the step size, and $\nabla$. is the divergence operator. $^{1}$

$$
\delta S_{n+1}=\delta S_{n}(1+\delta l \nabla \cdot \vec{F})+O\left(\delta l^{2}\right)
$$

The complete advection path of $\delta S$ conceptually forms a tube, whose cross-sectional area represents the local squeezing of cortical columns. Successive values of $\delta S$ can be integrated along the path, yielding the volume of the tube. As each cortical voxel is advected towards both surfaces, it is associated

\footnotetext{
${ }^{1}$ For demonstrating (4), consider a small portion of cone comprised between the elementary surfaces $\delta S_{n}$ and $\delta S_{n+1}$. The divergence theorem gives the relationship between $\delta S_{n}, \delta S_{n+1}$, and $\nabla \cdot \vec{F}$, considering that $\|\vec{F}\|=1$ and that the integral on the cone's lateral surface is zero because this surface is tangent to $\vec{F}$.
} 


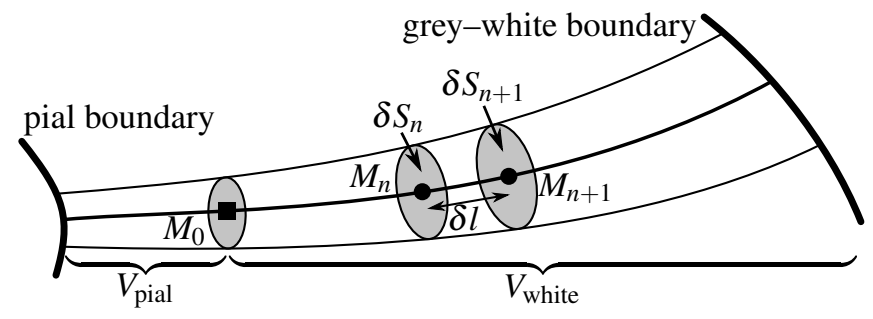

Fig. 2: The advection of an elementary surface along $\vec{F}$ conceptually models the shape of a cortical column. The volume of the tube on either side of $M$ is obtained by integrating $\delta S$ along the advection path.

with two volumes $V_{\text {pial }}$ and $V_{\text {white. }}$ The relative equivolumic depth can then be defined as

$$
d_{\mathrm{vol}}=\frac{V_{\text {pial }}}{V_{\text {pial }}+V_{\text {white }}} .
$$

The implementation of this method is made available as free software [7].

\subsection{Metrics used for comparison}

Two other metrics are defined to serve as references for evaluation of the equivolumic metric.

The Euclidean metric is often used in slice-by-slice analysis of histological images. State-of-the-art implementations [3] sample automatically generated Laplacian traverses with a fixed number of evenly spaced measurements. This metric was implemented in the following way, so as to produce a depth value for each cortical voxel: the geodesic Euclidean distances to the pial surface $\left(d_{\text {pial }}\right)$ and grey-white surface $\left(d_{\text {white }}\right)$ were measured along Laplacian traverses built from a 3D segmentation, using an advection procedure similar to that described in $\S 3.2$. The Euclidean metric is then defined by (6).

$$
d_{\text {Eucl }}=\frac{d_{\text {pial }}}{d_{\text {pial }}+d_{\text {white }}}
$$

Using the raw value $T$ of the Laplacian field for measuring depth amounts to defining cortical layers as isocontours of $T$, which are by definition orthogonal to $\vec{F}$. In other words, this model of cortical layers stems from the hypothesis that layers are orthogonal to columns everywhere in the cortex. This model has been used previously as a reference for comparison [5]. Although other studies that advertised using a "Laplace model" were in fact using the previously introduced Euclidean metric along Laplacian traverses, this model was tested because it allows to challenge the orthogonality of columns and layers.

\section{RESULTS}

The proposed framework was tested on a magnetic resonance image of a post-mortem human brain. Post-mortem tissue was

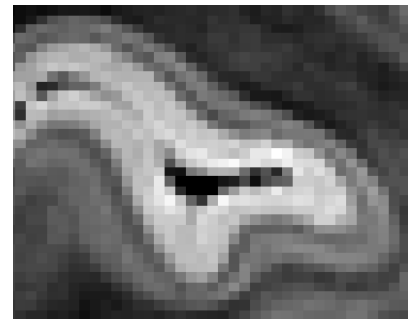

(a) Raw MR image

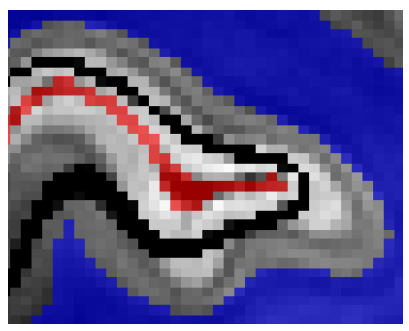

(c) Laplacian field $T$

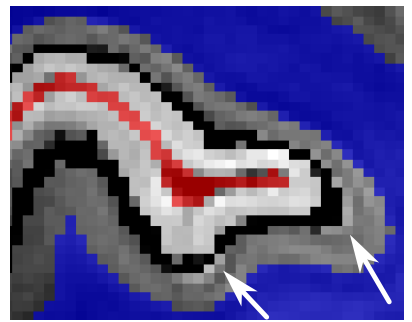

(b) Euclidean $d_{\text {Eucl }}$

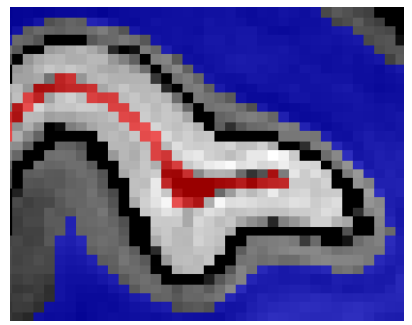

(d) Equivolumic $d_{\text {vol }}$
Fig. 3: Depth isocontours centred on $d=0.5$ for different metrics are overlaid in black on a coronal slice showing the calcarine fissure. The segmentation is overlaid, with white matter in blue, CSF in red.

used for this pilot study because it allows to reach a higher resolution than in vivo, thanks to long acquisition times. In vivo protocols can be designed to provide similar contrast.

The image was acquired on a human $7 \mathrm{~T} \mathrm{MRI} \mathrm{system,} \mathrm{with}$ a resolution of $0.31 \times 0.31 \times 0.35 \mathrm{~mm}^{3}$, using a variable flip angle turbo spin echo sequence (SPACE) yielding $\mathrm{T}_{2}$-weighted contrast (acquisition time $1 \mathrm{~h} 48 \mathrm{~min}$.).

A three-compartment segmentation (GM, WM, CSF) of the left occipital lobe was constructed largely by hand, with assistance from tools of the Morphologist segmentation pipeline [8] for obtaining the correct topology.

Figures 3 and 4 compare the proposed equivolumic metric with the reference metrics introduced in $\S 3.3$. The depth of every cortical voxel is calculated according to each metric. The comparison is done within the calcarine fissure, which is a good region to test depth metrics because its cortex is very convoluted and has a strong lamination pattern, with a distinctive layer IV called the stria of Gennari.

The Euclidean layer represented on Fig. 3 is equidistant from both cortical surfaces. This confirms that the stria of Gennari is pushed away of the regions of high curvature such as sulcal fundi (arrows). The Laplacian field $T$ follows layers even less accurately than the Euclidean metric, because it has a tendency to "cut corners", leading to very thin layers in regions of high curvature. The equivolumic metric is very consistent with the stria of Gennari.

Figure 4 represents lamination profiles as scatter plots, where each voxel is represented by its depth and intensity. 


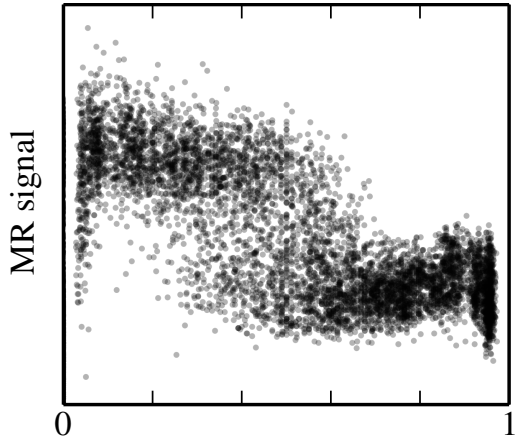

(a) Euclidean $d_{\text {Eucl }}$

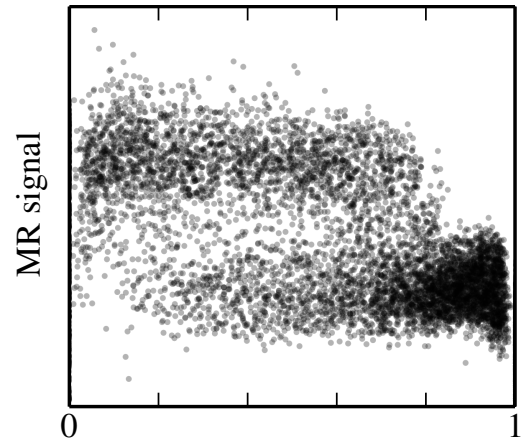

(b) Laplacian field $T$

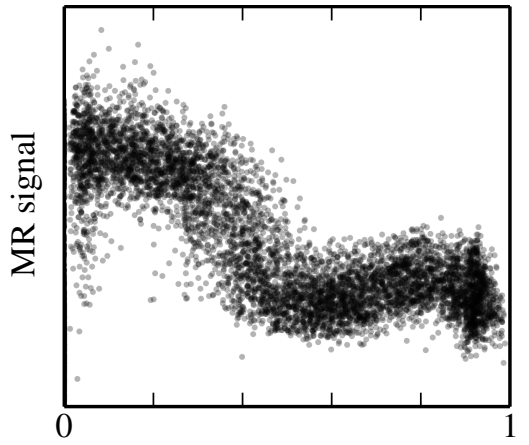

(c) Equivolumic $d_{\mathrm{vol}}$

Fig. 4: Lamination profiles extracted in a large portion of the calcarine fissure that covers sulci and buried gyri. The proposed equivolumic metric yields the most informative lamination profile, because it introduces the least dispersion.

These profiles represent a large $\left(278 \mathrm{~mm}^{3}\right)$, manually segmented 3D region of the calcarine fissure, which was chosen to contain both positive and negative curvatures (sulci and buried gyri). The lamination pattern is revealed most clearly with the equivolumic metric, while other metrics introduce dispersion which blurs the profile.

\section{CONCLUSION AND PERSPECTIVES}

The results confirm that the equivolumic metric compensates cortical curvature best. Compared to the previously described method [5], the proposed formalism allows precise modelbased estimation of the shape of cortical columns, rather than relying on an interpolated approximation of the curvature. Moreover, it integrates the modelling of columns and layers in a consistent framework. Combined with automatic generation of volumetric cortical traverses [9], this framework can be used to extract lamination profiles that cover the cortical surface with regular spacing, and preserve the original voxel sampling by avoiding the need to interpolate the data at non-integer coordinates.

Insofar as cortical columns seem to follow the gradient $\vec{F}$ of the Laplacian field $T$ (see [3], or Fig. 1), it is interesting to note that cortical layers do not follow equipotentials of $T$. This suggests that the orthogonality of columns and layers does not hold in the curved cortex. While further work will be needed to properly validate the Laplacian model of columns, this obviates the need for complementary, but distinct, models of cortical columns and layers.

\section{ACKNOWLEDGEMENTS}

Many thanks go to Benoît Schmitt, Alexandre Vignaud, and Christine Delmaire, who took part in the acquisition of the MR image that was used to demonstrate this framework, and to Caroline Jan, who produced the histological data.

\section{REFERENCES}

[1] S. E. Jones et al., "Three-dimensional mapping of cortical thickness using Laplace's equation," Human Brain Mapping, vol. 11, pp. 12-32, 2000.

[2] O. Schmitt and M. Böhme, "A robust transcortical profile scanner for generating 2-D traverses in histological sections of richly curved cortical courses," NeuroImage, vol. 16, no. 4, pp. 1103-1119, Aug. 2002.

[3] A. Schleicher et al., "Quantitative architectural analysis: a new approach to cortical mapping," Anatomy and Embryology, vol. 210, no. 5-6, pp. 373-386, Dec. 2005.

[4] J. Dauguet et al., "Three-dimensional reconstruction of stained histological slices and 3d non-linear registration with in-vivo MRI for whole baboon brain," Journal of Neuroscience Methods, vol. 164, no. 1, pp. 191-204, Aug. 2007.

[5] M. D. Waehnert et al., "Anatomically motivated modeling of cortical laminae," NeuroImage, vol. 93, pp. 210-220, June 2014.

[6] S. T. Bok, "Der Einfluß der in den Furchen und Windungen auftretenden Krümmungen der Großhirnrinde auf die Rindenarchitektur," Zeitschrift für die gesamte Neurologie und Psychiatrie, vol. 121, no. 1, pp. 682-750, Dec. 1929.

[7] Y. Leprince et al., "highres-cortex: analysis tools for high resolution cortical MRI," github.com/neurospin/ highres-cortex, 2014.

[8] "Morphologist: T1 MRI segmentation, cortical sulci recognition and morphometry toolbox," www.brainvisa.info.

[9] Y. Leprince et al., "Architectonics-informed partition of the cortex at sub-millimetre resolution," in 20th Annual Meeting of OHBM, Hamburg, Germany, June 2014, hal-cea.archives-ouvertes.fr/cea-01074735. 